Garcés-Delgado, M., Santana-Vega, L. E. y Feliciano-García, L. (2020). Proyectos de vida en adolescentes en riesgo de exclusión social. Revista de Investigación Educativa, 38(1), 149-165.

DOI: http://dx.doi.org/10.6018/rie.332231

\title{
Proyectos de vida en adolescentes en riesgo de exclusión social
}

\author{
Life designs in adolescents at risk of social exclusion \\ Yaritza Garcés-Delgado, Lidia E. Santana-Vega y Luis Feliciano-García \\ Departamento de Didáctica e Investigación Educativa \\ Facultad de Educación. Universidad de La Laguna (España)
}

\begin{abstract}
Resumen
La configuración del proyecto de vida es un reto de todo ser humano. Los factores que condicionan la construcción del proyecto vital son diversos: escasa madurez, falta de identidad, personalidad conflictiva, entorno sociofamiliar problemático, fracaso escolar, etc. La investigación tiene como objetivo analizar el proceso de configuración de los proyectos de vida en adolescentes en riesgo de exclusión social. Se realizó un estudio de casos múltiples con seis adolescentes con trayectorias de exclusión acogidos al sistema de protección. En el estudio se utilizaron instrumentos y técnicas de recogida de información cualitativas (entrevistas semiestructuradas y diario de campo) y cuantitativas (cuestionario) optando por una opción de metodología mixta. Los resultados muestran que estos menores poseen dificultades para proyectar un futuro al margen de los contextos de exclusión social en los que se han desarrollado. Los proyectos de vida son construidos desde la inadaptación al sistema y sobre una base socioemocional deficiente, ocasionando la incapacidad de generar pensamientos y conductas asertivas para lograr alcanzar las metas fijadas. Es necesario: a) trabajar con los adolescentes en riesgo de exclusión la adquisición de estrategias socioemocionales, y b) prevenir las conductas intra e interpersonales conflictivas, a través de acciones de atención/apoyo.

Palabras clave: adolescencia; discriminación social; proyecto de vida; estudio de casos; toma de decisión.
\end{abstract}

Correspondencia: Yaritza Garcés-Delgado, ygarcesd@ull.edu.es, Av. Trinidad s/n. Campus Central. 38204, San Cristóbal de La Laguna. Tenerife. España. Tfno. 0034-922319220. 


\begin{abstract}
The configuration of the life design is a challenge for every human being. The factors that determine the construction of the life design are diverse: lack of maturity, lack of identity, conflictive personality, problematic social and family environment, school failure, etc. This study attempts to analyse the process of shaping life designs in adolescents at risk of social exclusion. A multi-case study was conducted with six adolescents presenting exclusion trajectories that were within the protection system. In the study, qualitative (semi-structured and diary field interviews) and quantitative (questionnaire) data retrieval tools and techniques were used, opting for a mixed methodology. The results show that these children have difficulties in projecting a future outside the contexts of social exclusion in which they have developed. Life designs are built from the maladjustment to the system and on a deficient social-emotional basis, making young people unable to generate assertive thoughts and behaviors to achieve the goals set. It is necessary to: a) work with adolescents at risk of exclusion to make them acquire social-emotional strategies, and b) prevent conflicting intra and interpersonal behaviour through care/support actions.

Keywords: adolescence; social discrimination; life design; case study; decision-making.
\end{abstract}

\title{
Introducción
}

Una sociedad concienciada, crítica y responsable es aquella educada y orientada para saber ser, saber hacer, saber relacionarse, saber sentir $y$, por ende, capaz de dotar a la ciudadanía de competencias necesarias para el desarrollo de proyectos vitales sólidos y satisfactorios, y ajustados a su realidad (Freire, 1998).

Los proyectos de vida son el resultado de la conciliación entre la toma de decisiones racional y emocional. La toma de decisiones no solo se debe a un proceso puramente cognitivo, sino que está fuertemente influenciada por las condiciones inter e intrapersonales de cada individuo: emociones, sentimientos, temperamento, intereses, etc. La capacidad de proyectar nuestra vida en el futuro permite a las personas elaborar metas y objetivos en el ámbito personal, social, cultural, laboral, moral, etc. (Lomelí-Parga, López-Padilla \& Valenzuela-González, 2016; Melendro, 2014; Pérez, Poza \& Fernández, 2016; Pérez \& Melendro, 2016; Rodríguez, Juanas \& González, 2016).

La adolescencia es el preludio a la vida adulta; en esta etapa se debe armonizar el "soy" (del presente) y el "querer ser" (del futuro) para la consecución de un proyecto vital satisfactorio. Según Boutinet (2002) hay tres tipos de proyectos centrados en la adolescencia: el escolar, el profesional y el de vida. La configuración del proyecto escolar y profesional propiciará el desarrollo de las capacidades personales, sociales y culturales, imprescindibles para la maduración. Sin embargo, el proyecto de vida es una meta a largo plazo; se trata del estilo de vida que un adolescente pretende adoptar en un futuro más o menos lejano.

Los adolescentes deben enfrentarse a periodos de grandes incertidumbres a la hora de tomar decisiones, valorando sus habilidades, competencias e intereses para la configuración de un proyecto vital razonado y adaptado a sus necesidades personales (Santana-Vega, 2015). Tener un proyecto vital sólido y viable es complicado, pero absolutamente necesario si se quiere manejar las complejidades de las trayectorias vitales y las transiciones relacionadas con la carrera (Maree, 2018). 
La adolescencia dentro de la exclusión es un proceso de injusticia social, de desigualdad de oportunidades y desventaja respecto al grupo de iguales (Melendro, Cruz, Iglesias \& Montserrat, 2014). Un adolescente en riesgo de exclusión social tendrá menos posibilidades de elaborar un proyecto de vida enfocado al crecimiento personal, social, afectivo y laboral que un adolescente que no esté en situación de riesgo (Parrilla, Gallego \& Moriña, 2010). Los proyectos de vida en la adolescencia pueden dotar a los jóvenes de felicidad y/o resiliencia en función de los fines logrados. Asimismo, la obtención de la felicidad es una de las finalidades de construir un proyecto vital; la vía para su logro es desarrollar todas las capacidades sociopersonales para mejorar la calidad de vida (Damon, 2009). La configuración del proyecto vital en la adolescencia sirve para enfocar ideas, pedir orientación y opiniones, solicitar apoyos institucionales, etc. (Caravantes, Climent \& Masiá, 2017; Castel, 2014; Ducca-Cisneros, 2018). Construir un proyecto de vida sería es la puerta de entrada a la inclusión social para los adolescentes en riesgo de exclusión social; salir o no de la trayectoria de exclusión dependerá de cómo afronten su situación, de sus apoyos, recursos, posibilidades y expectativas (Ballester, Caride, Melendro \& Montserrat, 2016; García, Quintanal \& Cuenca, 2016; Melendro, de Juanas \& Rodríguez, 2017; Parrilla et al., 2010; Pérez et al., 2016, Santana, Alonso \& Feliciano, 2018).

En la adolescencia, la creación de este tipo de proyectos es un pasaje hacia la estabilidad emocional y social, pues todo ser humano necesita guiarse y encontrarse a sí mismo. Un adolescente en una situación de vulnerabilidad social debe asumir con resiliencia dos factores: 1) los cambios propios de la adolescencia y 2) su situación de desventaja social. Cuando la vida se desarrollada en un contexto inapropiado y de exclusión deben buscarse estrategias de supervivencia. Según Parrilla et al. (2010), un joven para afrontar su proyecto vital y tomar decisiones vocacionales correctas debe: a) conocer e identificar todas sus cualidades y motivaciones, b) ser consciente de sus aptitudes y actitudes, y c) identificar su personalidad. Todos estos elementos interfieren tanto en la adquisición de habilidades sociales como en el desarrollo emocional y madurativo de las personas.

Según Bendit \& Hahn-Bleibtreu (2008) las barreras sociales y estructurales a las que se enfrenta un joven en riesgo de exclusión social suponen un hándicap en su integración, adaptación y participación en la sociedad. Varios estudios explican y describen las "trayectorias fallidas" de colectivos socialmente desfavorecidos (Ballester et al., 2016; BautistaCerro \& Melendro, 2011; García et al. 2016; Melendro, 2011; Melendro et al., 2014; Pérez et al., 2016), mientras otros plantean la necesidad de reestructurar las políticas sociales de atención destinadas a estos colectivos (Bendit \& Stokes, 2004; Du Bois-Reymond \& López, 2004; Melendro, 2014; Melendro et al., 2017).

En este estudio el término "menores en riesgo de exclusión social" hace referencia a aquellos adolescentes que: 1) nacen y se desarrollan en familias de bajo nivel educativo y económico, 2) con escasas redes sociales de apoyo, 3) pertenecientes a culturas minoritarias excluidas o autoexcluidas socialmente, y 4) con desestructuración familiar y/o personal (Velaz de Medrano, 2005). Los elementos sociales y personales que intervienen en el proceso de desarrollo y crecimiento personal son determinantes para afrontar con solvencia y valentía los múltiples obstáculos a los que se enfrentan los jóvenes en riesgo de exclusión (González-Cubillán, 2009; Melendro, 2011; Melendro et al., 2014). Por todo ello, se considera necesario investigar sobre esta realidad y ofrecer posibles vías de estudio o de intervención bajo una dimensión de transformación socioeducativa. 


\section{Método}

Para desarrollar la investigación se parte de un estudio de casos múltiples. Este tipo de método permite desarrollar, describir y explicar los fines de la investigación de una forma detallada y rigurosa. Con el fin de obtener la información necesaria se utilizaron instrumentos y técnicas cualitativas y cuantitativas, en base a las necesidades que surgieron en cada momento de la investigación. El empleo de un enfoque mixto, combinando diversos tipos de técnicas e instrumentos, posibilita triangular la información aportada por los participantes (Anguera, Blanco-Villaseñor, Losada, Sánchez-Algarra y Onwuegbuzie, 2018).

\section{Objetivos}

El propósito principal de este estudio es analizar cómo las variables contextuales inciden en el proceso de construcción del proyecto vital de adolescentes en riesgo de exclusión. Los objetivos específicos son: a) analizar el proyecto de vida y las expectativas familiares percibidas por los adolescentes en riesgo de exclusión; b) identificar los factores que afectan a la construcción de sus proyectos vitales.

\section{Población y Muestra}

Seis casos fueron seleccionados de forma no aleatoria e intencional, según los criterios de la Tabla 1, con el consentimiento informado de los tutores legales y de los profesionales del centro de día ATAJO.

Tabla 1

Características de la muestra

\begin{tabular}{|c|c|c|c|c|c|c|c|}
\hline \multirow{2}{*}{$\begin{array}{l}\text { Criterios } \\
N^{o} d e \\
\text { casos }\end{array}$} & \multirow{2}{*}{$\begin{array}{c}\text { Características } \\
\text { generales }\end{array}$} & \multicolumn{6}{|c|}{$\begin{array}{c}\text { Características } \\
\text { particulares }\end{array}$} \\
\hline & & Caso 1 & Caso 2 & Caso 3 & Caso 4 & Caso 5 & Caso 6 \\
\hline Sexo & equitativo & Mujer & Mujer & Hombre & Hombre & Hombre & Mujer \\
\hline Edades & 16-17 años & $\begin{array}{c}17 \\
\text { ACAD }\end{array}$ & $\begin{array}{c}17 \\
\mathrm{ACAD}\end{array}$ & $\begin{array}{c}17 \\
\text { ACAD }\end{array}$ & $\begin{array}{c}17 \\
\text { ACAD }\end{array}$ & $\begin{array}{c}16 \\
\text { ACAD }\end{array}$ & $\begin{array}{c}16 \\
\mathrm{ACAD}\end{array}$ \\
\hline Factor de & Factores & $\begin{array}{l}\text { CONT } \\
\text { FAMI }\end{array}$ & CONT & CONT & CONT & $\begin{array}{l}\text { CONT } \\
\text { FAMI }\end{array}$ & CONT \\
\hline $\begin{array}{l}\text { exclusión } \\
\text { social }\end{array}$ & $\begin{array}{c}\text { Varios } \\
\left(^{*}\right)\end{array}$ & & & $\begin{array}{l}\text { FAMI } \\
\text { DELI }\end{array}$ & AUTO & BULL & \\
\hline & & & & DROG & & & \\
\hline
\end{tabular}




\begin{tabular}{|c|c|c|c|c|c|c|c|}
\hline Criterios & $\begin{array}{c}\text { Características } \\
\text { generales }\end{array}$ & & & $\begin{array}{r}\text { Car } \\
\text { pa }\end{array}$ & $\begin{array}{l}\text { sticas } \\
\text { lares }\end{array}$ & & \\
\hline Centro & Todos & Atajo & Atajo & Atajo & Atajo & Atajo & Atajo \\
\hline de Día & & & & Tafira & & & \\
\hline
\end{tabular}

ACAD: académicos; FAMI: familiares; DELI: delictivos; DROG: drogadicción; CONT: contexto; BULL: bullying; AUTO: autoexclusión

Los seis casos se encuentran en riesgo de exclusión social por motivos diferentes, siendo comunes la frustración y el fracaso escolar y el entorno de deprivación social en el que viven. El criterio de heterogeneidad de los estudios de casos múltiples se cumple con las singularidades que presenta cada adolescente. También participó en el estudio una de las educadoras del centro de día al que pertenecen los menores. El fin de dicha participación era la obtención y contraste de la información ofrecida por los menores.

\section{Instrumentos y técnicas}

Entrevista semi-estructurada a adolescentes [E-AD]. La entrevista es una de las técnicas de investigación cualitativa más potente, junto con la observación, que se pueden utilizar en un estudio científico de carácter socioeducativo. Este estudio permitió un acercamiento personalizado a los adolescentes para profundizar sobre: situación familiar, entorno social, conocimiento/regulación de las emociones propias, metas personales, expectativas académico-laborales, expectativas familiares percibidas, habilidades sociales, etc. La entrevista se realizó según el siguiente el guion: a) Problemática del ambiente social, familiar, educativo y personal; b) Metas u objetivos personales; c) Toma de decisiones; d) Expectativas familiares.

Entrevista semi-estructurada a la educadora [E-ED]. Las cuestiones del guión de esta entrevista estaban relacionadas con los temas abordados en la entrevista de los adolescentes con el fin contrastar la información aportada por estos.

Diario de campo [DC]. Esta técnica se utilizó para recoger información sobre cada una de las sesiones realizadas en el campo de estudio, permitiendo complementar información sobre los casos analizados.

Cuestionario de Orientación Académica y Laboral [COAL]. El COAL se utilizó para explorar las ideas, creencias y opiniones de los casos sobre sus proyectos personales de vida. Se realizó una adaptación del Cuestionario de Orientación Académica y Laboral, diseñado por el Grupo de Investigación y Orientación Educativa y Sociolaboral (GIOES) de la Universidad de La Laguna. En la Tabla 2 se presentan las dimensiones de información, las preguntas, los ítems y el $\alpha$ de Cronbach de las escalas incluidas en el cuestionario. 
Tabla 2

Descripción del COAL

\begin{tabular}{lccc}
\hline Dimensiones & Pregunta & Ítems & $\alpha$ de Cronbach \\
\hline Información personal & $1 ; 2 ; 3$ & 3 & - \\
Expectativas académico-laborales & $4 ; 5$ & 2 & - \\
Habilidades personales & 7 & 17 & 0.72 \\
Expectativas y apoyo familiar afectivo percibido & $6 ; 8$ & 5 & 0.81 \\
Metas del proyecto de vida & 9 & 33 & 0.81 \\
Madurez vocacional & 10 & 14 & 0.94 \\
Autoeficacia vocacional & 11 & 13 & 0.72 \\
Claridad del proyecto personal de vida & 12 & 4 & \\
\hline
\end{tabular}

La escala de Figuera, Dorio y Forner (2003) se aplicó para analizar el apoyo familiar afectivo percibido; el índice de consistencia interna obtenido fue de 0.72 (SantanaVega, Feliciano-García \& Jiménez, 2016). Se utilizó una adaptación del Cuestionario de Madurez de la Carrera de Álvarez, Bisquerra, Espín y Rodríguez (2007) para analizar la madurez vocacional; el índice de consistencia interna obtenido fue de 0.81 (Santana-Vega, Feliciano-García \& Santana, 2013). La escala de Eficacia en la Toma de decisiones de Carbonero y Merino (2003) se empleó para examinar la autoeficacia vocacional; el índice de consistencia interna obtenido fue de 0.94 (Santana-Vega et al., 2013). Se diseñó una escala ad hoc de 4 ítems para analizar la claridad del proyecto de vida; el índice de consistencia interna obtenido fue de 0.72 (Santana-Vega et al., 2013). Finalmente, para analizar las metas del proyecto de vida se diseñó ad hoc una escala en la que se examina el grado de prioridad que para los sujetos tienen 33 metas académicas, personales y profesionales; el índice de consistencia interna obtenido fue de 0.81 (Santana-Vega et al., 2016).

\section{Procedimiento de recogida y análisis de datos}

El proceso de recogida y análisis de los datos se realizó simultáneamente a lo largo de un año. El diario de campo se redactó in situ y a posteriori de las visitas al Centro de Día. El COAL fue cumplimentado individualmente y en diferentes días, su cumplimentación osciló entre los 15 y 20 minutos. Las entrevistas fueron realizadas a cada menor en días y semanas diferentes, la duración aproximada de las entrevistas osciló entre los 40 y 50 minutos, dependiendo de la fluidez y contenido de las respuestas. Finalmente, se llevó a cabo la entrevista con la educadora del centro en dos sesiones de 37 y 48 minutos. 
Tabla 3

Procedimiento de recogida de datos

\begin{tabular}{lccc}
\hline Técnica & Temporalidad & № sesiones & Duración \\
\hline Entrevistas & 2 meses & 16 sesiones \\
& 3 días & 2 sesiones por adolescente, & $40-45$ min. \\
Cuestionario & 1 año & 3 sesiones la educadora & \\
Diario de campo & & 144 sesiones & $\begin{array}{c}15-20 \text { min. } \\
\text { horas, } 3 \text { veces por } \\
\text { semana }\end{array}$ \\
\hline
\end{tabular}

Los datos obtenidos por medio del COAL se analizaron obteniendo las puntuaciones directas de cada dimensión, posteriormente se realizó un perfil para interpretar los datos obtenidos de forma cualitativa (utilizándose el mismo sistema de codificación que las entrevistas), por lo que se realizaron descripciones cualitativas de los resultados cuantitativos. El análisis de las E-AD, las E-ED y el DC fue un procedimiento iterativo que implicó introducir los datos en el programa informático ATLAS.ti. Este procedimiento implicó la transcripción, codificación, categorización, definición de las categorías y recodificación de las "familias"; en ATLAS.ti las familias son las categorías temáticas (Saldaña, 2009).

Tabla 4

Sistema de codificación del análisis cualitativo

\begin{tabular}{cccc}
\hline Dimensión & Categorías & Temas & Códigos \\
\hline & $\begin{array}{c}\text { Dificultades en el am- } \\
\text { biente social, familiar, } \\
\text { educativo y personal }\end{array}$ & Dificultades familiares & Fracaso escolar \\
\cline { 2 - 4 } & Conflictos sociopersonales & PPV-DIF. G \\
\cline { 2 - 4 } $\begin{array}{c}\text { Construcción de los } \\
\text { proyectos personales } \\
\text { de vida }\end{array}$ & personales & Corto plazo & PPV-MET/OB \\
\cline { 2 - 4 } & Toma de decisiones & Targo plazo & Tiabilidad \\
& & Racionamiento de decisiones & PPV-T.DEC \\
\cline { 2 - 4 } & Opinión familiar & Preocupación familiar & PPV-OP.FAM \\
& & Influencia familiar & \\
\hline
\end{tabular}


Para asegurar la credibilidad de los datos se implementaron las siguientes estrategias: a) triangulación de fuentes y técnicas y b) Contrastación de la información con los participantes.

\section{Resultados}

A continuación se presentan los resultados de la información obtenida a través de las entrevistas semi-estructuradas (a los adolescentes y la educadora), el cuestionario y el diario de campo. La presentación de los resultados se estructura en torno a las dimensiones del cuestionario (Expectativas académico-laborales; Habilidades personales; Expectativas y apoyo familiar afectivo percibido; Metas del proyecto de vida; Madurez vocacional; Autoeficacia vocacional; Claridad del proyecto personal de vida)

\section{Contextualización}

Los casos analizados en este estudio pertenecen a barrios con un alto índice de menores en riesgo de exclusión social (La Feria, Vega de San José y Las Rehoyas). El Centro de Día Atajo se encuentra ubicado en el polígono de La Vega de San José al noreste del municipio de Las Palmas de Gran Canaria. El barrio de la Vega de San José se caracteriza por tener un importante número de viviendas sociales. Los datos revelan que el estatus socioeconómico de las familias que habitan en el polígono es bajo:

El Centro ATAJO está dentro de un barrio que a la vista solo tiene viviendas sociales, denominadas comúnmente como barriadas, en un estado de deterioro importante y ordenadas en forma rectangular alrededor del Centro de Día y del Colegio de Educación de Infantil y Primaria de la zona. El Centro está situado en un lugar vulnerable. En ese contexto tan complejo las acciones que realice ATAJO pueden ayudar a los jóvenes a suplir las carencias del entorno [DC-PPV-DIF. G4].

El Centro de Día ATAJO pertenece a la Fundación ADSIS, una Organización No Gubernamental que realiza actividades enfocadas al desarrollo personal y al equilibrio social. Las acciones realizadas desde la Fundación centran su interés en el desarrollo integral de las personas; concretamente de grupos empobrecidos y/o excluidos. Según la educadora del Centro, los factores que ponen en riesgo de exclusión a los jóvenes son los siguientes:

El momento de la adolescencia, el idealizar a referentes que no son los recomendados, viéndolos con normalidad y sabiendo que están pendientes de un hilo, sus propias familias en muchos casos que, aunque les apoyan, han vivido esa realidad $y$, también, lo que han sufrido [E-ED-PPV-DIF.G5].

Cuando un adolescente está expuesto a situaciones límites puede generar actitudes de rechazo, frustración, estrés y ansiedad. En los Casos 1, 2 y 6 la situación de precariedad del contexto sociofamiliar y las características personales de las adolescentes 
han generado "trayectorias fallidas" en el ámbito escolar, familiar, personal y social. El Caso 3 ha vivido desde su infancia en distintos centros para menores debido a su conducta, a los conflictos familiares y al consumo y venta de drogas. En el caso 4 la desestructuración del seno familiar, el entorno próximo, las relaciones entre iguales, las drogas y el abandono escolar le hacen socialmente vulnerable. El Caso 5 ha sido víctima de acoso escolar y familiar a lo largo de la infancia y adolescencia.

\section{Expectativas académico-laborales}

En base a las respuestas obtenidas en el COAL, las expectativas académicas de los adolescentes se centran en la continuación de sus estudios y consecución de un título educativo; cursando los Programas de Cualificación Profesional Inicial (PCPI), la Educación Secundaria Obligatoria (ESO) o el Bachillerato. Respecto a sus expectativas laborales la mayoría se inclina por un trabajo en el sector servicios.

La joven del caso 1, parece tener claras las metas a corto plazo. Según los resultados del cuestionario, el afán por obtener estabilidad económica y personal ha hecho que se plantee los siguientes objetivos: a) sacar el graduado, b) hacer un curso de peluquería y c) lograr un trabajo estable. La adolescente del caso 2, visualiza como meta a corto plazo sacar el graduado y poder trabajar cuanto antes. Sus expectativas académicas y profesionales están unidas: "Mi único objetivo es sacarme el graduado solo para poder conseguir trabajo [...]. Me he dado cuenta de que sin el graduado no eres nadie. Me lo quiero sacar porque quiero trabajar y porque quiero aprender algo más" [E-ADPPV-MET/OB4].

El joven del caso 3 plantea entre sus metas prioritarias:

Aprender a leer más rápido y aprender a escribir mejor. Tener mi graduado, sacarme un PCPI [...]. Tener algo para el día de mañana porque si no...sin un trabajo no vas a hacer nada. Te vas a ver en la calle tirado [E-AD-PPV-MET/OB8].

Las metas que tantea el caso 4 son finalizar el graduado escolar y, en un futuro, seguir estudiando Bachillerato. Le gustaría ser Guardia Civil, empresario o abogado. El caso 5 quiere estudiar hasta el Bachillerato, le gustaría ser actor, camarero o profesor de actividades extraescolares.

La joven del caso 6 es la única que abiertamente expresa rechazo a los estudios: "Bueno, en lo académico no saco nada porque no me pongo a estudiar, es que no puedo, bueno si puedo, pero no quiero. Odio estudiar" [E-AD-PPV-MET/OB14]. Sin embargo, una de sus metas a corto plazo es obtener el graduado escolar.

Los menores han vivido realidades complicadas a lo largo de su desarrollo, algunos las han interiorizado (Caso 1, 2, 4 y 6) y otros luchan por cambiarla (Casos 3 y 5). Planificar un proyecto de vida cobra sentido cuando las capacidades emocionales, sociales, educativas y profesionales se encuentran en armonía. La educadora explica que algunos jóvenes tomaron decisiones equívocas en el ámbito académico: "La mayoría de ellos no siguen en el instituto porque no han tomado decisiones correctas. Han sido expulsados por el propio instituto debido a sus conductas. El carácter influye negativamente en sus decisiones" [E-ED-PPV-T.DEC2]. 


\section{Habilidades personales}

Teniendo en cuenta la información ofrecida por los adolescentes en las entrevistas, se constata que algunos presentan dificultades para reconocer sus equivocaciones y para transmitir sus ideas a los demás. Todos admiten que sus objetivos son poco viables e irreales; además manifiestan dificultades para ser constantes en lo que se proponen. Específicamente, según los datos del COAL, los casos 1, 3, 4, 5 y 6 se valoran a sí mismos como: 1) incapaces de solucionar sus problemas de forma solvente y asertiva; 2) sin iniciativa para desarrollar sus propias ideas y 3) con problemas para organizar su tiempo. Son conscientes de no haber hecho las cosas bien a lo largo de su vida; y señalan su incapacidad para negarse ante propuestas que les perjudican (drogas, delincuencia, alcohol, etc.). En cuanto al caso 2, se percibe a sí misma como una persona sociable y consecuente con sus actos, no obstante, destaca su dificultad para admitir sus equivocaciones.

\section{Expectativas y apoyo familiar afectivo percibido}

Las respuestas ofrecidas en el cuestionario muestran que los familiares de estos adolescentes prefieren que comiencen a trabajar cuándo hayan finalizado sus estudios. Las argumentaciones de los casos estudiados son determinantes para entender las expectativas de sus familiares:

La adolescente del caso 1 expone:

Ellos me dicen que estudie, que lo primero es el graduado y después el trabajo, que no tenga prisa. Me dicen que puedo tener un buen trabajo como policía. Aunque también me apoyan con lo de ser mi propia jefa, pero me lo dicen más bien para que no dependa de nadie. También les gustaría que me casara [E-AD-PPV-OP.FAM9].

Los jóvenes del estudio consideran que sus familiares tienen expectativas educativas y laborales mayores a las que ellos creen que pueden optar. Según el caso 2: "Ellos quieren que me saque el graduado. Bueno, mis padres y toda mi familia quieren que siga estudiando. Ellos no querían que yo dejara el instituto y pensaban en que podía llegar a la Universidad, pero ya ves..." [E-AD-PPV-OP.FAM12].

El joven del caso 3 explica: "Mi padre lo único que quiere es que tenga un trabajo el día de mañana y que tenga el graduado. En mi familia solo lo tienen dos personas" [E-AD-PPVOP.FAM16]. El caso 4 reconoce que sus familiares han decidido inscribirlo en el Centro de Día para que obtenga los conocimientos mínimos y pueda cursar el Bachillerato:

Ellos si pudieran elegir, elegirían que yo estuviese estudiando, ahora mismo debería estar haciendo $1^{o}$ de Bachiller, pero repetí. Apoyan totalmente que siga estudiando y deje de hacer el "gamberro". Me metieron aquí para no estar buscándome trabajo y para tener los conceptos básicos y el año que viene estudiar [E-AD-PPV-OP.FAM17].

Algunos menores consideran que sus expectativas laborales no coinciden con las de sus familiares. En este sentido, el joven del caso 5 comenta: "No me apoyan y me 
desaniman totalmente. [...]La verdad es que casi todo lo que yo quiero, ellos, lo ven negativo; sobre todo mi madre" [E-AD-PPV-OP.FAM19]. La joven del caso 6 manifiesta: "[...] si no hago nada me echan de mi casa, ya estoy avisada. Ellos prefieren que estudie antes que trabaje. [...] siempre me han dejado hacer lo que quiero" [E-AD-PPV-OP.FAM21].

Los progenitores de estos adolescentes apoyan la continuidad de los estudios como vía de escape para que sus hijos salgan de los procesos de exclusión y marginalidad. Difícilmente a estas edades, y sin haber conocido otros modelos socioeducativos, podrán plantearse la configuración del proyecto vital al margen de sus contextos sociofamiliares.

\section{Metas del proyecto de vida}

Conforme a las respuestas ofrecidas en el cuestionario, para estos adolescentes los objetivos prioritarios de su proyecto vital se centran en el ocio y tiempo libre, en tener dinero y en mudarse del barrio donde viven. Además, consideran relevante tener buena relación con sus padres y encontrar un trabajo que les ayude a tener estabilidad económica para formar una familia. No ven prioritario realizar una carrera o estudiar en el extranjero (menos el caso 5, que sí pretende salir de España).

Respecto a las metas del proyecto vital, el caso 1 y 4 coinciden: "Mis prioridades actuales son seguir estudiando, tener buena relación con mis padres y disfrutar del ocio y tiempo libre" [E-AD-PPV-MET/OB1]. "Mis metas de vida son finalizar los estudios, llevarme bien con mis padres y disfrutar del ocio" [E-AD-PPV-MET/OB11]. La joven del caso 2 relata: "Deseo pasar la mayoría del tiempo con mi novio. Para mí es prioritario mantener buena relación con mis padres, encontrar un trabajo pronto y ganar dinero" [E-AD-PPV-MET/OB6].

El menor del caso 3 expresa su deseo por formar una familia: "Mi mayor ilusión es formar una familia con mi actual pareja; quiero tener una familia en donde trabajemos los dos. Pero prefiero trabajar yo antes que ella para que esté con el niño y atienda la casa" [E-AD-PPVMET/OB7]. En este sentido, el caso 5 argumenta: "Mis objetivos son tener una familia e irme a vivir al extranjero solo. Me gustaría ser padre joven, pero si no tengo trabajo no puedo mantener a mi familia" [E-AD-PPV-MET/OB13].

La joven del caso 6 es la única en reconocer que sus metas son ilusiones y no realidades: "Realmente, no me he propuesto ni metas de esas a corto ni a largo plazo, lo que digo es lo que me gustaría tener, pero no hago nada por conseguirlo, simplemente vivo el momento" [E-AD-PPV-MET/OB18].

\section{Madurez vocacional}

De acuerdo con las respuestas ofrecidas por los adolescentes en los ítems de la escala de madurez vocacional, la mayoría reconocen que cambian continuamente sus decisiones vocacionales y que se dejan influenciar en sus decisiones. Además, consideran que acceder al mercado laboral es cuestión de suerte y/o amistad. Solo dos adolescentes afirman que no se dejan influenciar por los demás en sus decisiones vocacionales.

La joven del caso 1 reconoce cuáles son sus prioridades actuales y sus expectativas de futuro: "Tengo claro qué profesión quiero desempeñar; nadie me hará cambiar de opinión. Actualmente no me preocupo por el trabajo que quiero desempeñar porque estoy centrada en terminar los estudios para luego hacer peluquería" [E-AD-PPV-T.DEC1]. Asimismo, 
la menor del caso 2 explica: "Considero que no me dejo influenciar en mis expectativas académicas y profesionales. Elegiré una profesión que me guste y no una que agrade a mis padres" [E-AD-PPV-T.DEC4].

Los casos 3, 5 y 6 confiesan tener dificultades para tomar decisiones; impidiéndoles lograr las metas que se proponen. Los menores de los casos 3 y 5 expresan opiniones similares: "Tengo problemas a la hora de tomar decisiones. La poca confianza que tengo en mí mismo y en la consecución de mis metas ha hecho que los demás influyan en mis decisiones" [E-AD-PPV-T.DEC3]. Respecto a la joven del caso 6, ésta reconoce: "Tomo decisiones equivocadas continuamente; si me dicen que no lo voy a conseguir [...] Directamente ni lo intento. Esto hace que me desinterese por los estudios y otros ámbitos de mi vida" [E-AD-PPV-T.DEC7].

El menor del caso 4 se percibe a sí mismo de la siguiente forma:

Me siento poco preparado para alcanzar todos mis objetivos. Mi mayor problema es la falta de constancia en las decisiones que tomo. Generalmente me dejo influenciar por la opinión de otros. No tengo prioridades en el ámbito personal. A veces dudo en las decisiones que tomo, me cuesta mucho pensar. Yo estudiaba, pero también tomaba cosas... (drogas) [E-AD-PPV-T.DEC5].

\section{Autoeficacia vocacional}

Respecto a las respuestas ofrecidas por los adolescentes en los ítems de la escala de autoeficacia vocacional, la mayoría desconoce cuáles son sus limitaciones; suelen retrasar las decisiones finales si éstas son difíciles; toman decisiones precipitadas y generalmente equivocadas; considera que para conseguir un trabajo la cualificación es menos importante que la suerte.

El caso 1 cuando habla sobre la toma de decisiones comenta los errores que ha cometido durante su vida. La joven expresa el remordimiento de conciencia que le supone no haberse esforzado lo suficiente en el ámbito académico: "Ahora me arrepiento de no haber estudiado en el instituto" [E-AD-PPV-T.DEC8]. Su fracaso en el ámbito académico es uno de los múltiples resultados del vago e impulsivo procesamiento racional que realiza a la hora de tomar decisiones. Ser capaz de ver el lado positivo de la vida y de las situaciones que vivencia no ha sido suficiente para que tome decisiones acertadas.

La joven del caso 2 toma decisiones de forma inflexible sin pararse a pensar sobre sus fracasos o sus éxitos: "Si tomo una decisión ya la dejo fija y no la cambio por nada. Yo lo intento siempre hasta conseguirlo y si fracaso lo vuelvo a intentar una y otra vez, me da igual" [E-AD-PPV-T.DEC6]. La joven explica que toma decisiones equivocadas continuamente, haciendo que dude de sí misma.

El menor del caso 3 considera que no tiene iniciativa para poner en práctica lo que piensa, desea o quiere:

Si no hubiera hecho el "gilipollas" como he hecho, ponerme a fumar droga y esas cosas, yo en el instituto hubiese sacado algo. Si no me hubiera fugado del instituto. Yo llegaba allí y me quedaba hasta la hora del recreo o no entraba y me iba al sur con el colega a trabajar [E-AD-PPV-T.DEC12]. 
Respecto al caso 4, éste aplaza las decisiones cuando son complejas. Suele arrepentirse de las decisiones que toma y mantiene pensamientos negativos. Se considera desastroso tomando cualquier tipo de decisión. El caso 5 siente inseguridad cuando toma decisiones; esto le ocasiona problemas consigo mismo a la hora de perseguir sus metas u objetivos. El joven tiende a pensar de manera pesimista, sus pensamientos determinan y modulan negativamente sus acciones. A pesar de sus múltiples equivocaciones, muestra iniciativas para lograr sus propósitos.

Finalmente, la joven del caso 6 considera que tomar decisiones es una tarea complicada. Generalmente duda de las decisiones que toma: "Primero lo dudo, y si lo hago bien pues bien y si no pues también. Aunque si fracaso me sienta mal" [E-AD-PPV-T.DEC15].

\section{Claridad del proyecto personal de vida}

En base a las respuestas ofrecidas por los adolescentes en la escala de claridad del proyecto vital, la mayoría no se ha parado a pensar lo que hará en el futuro, sus proyecto son confusos, y planificar su futuro les resulta problemático. Quienes tienen claro su proyecto no han puesto los medios para alcanzar y materializar sus metas.

El caso 1 comenta que tiene claro y meditado su proyecto personal de vida. Pensar en su futuro no supone ningún tipo de esfuerzo ni dificultad. Sin embargo, le falta constancia y compromiso cuando ha decidido avanzar hacia una meta. Confiesa tener la capacidad de sobreponerse ante sus propios fracasos, añadiendo: "si dejo a medias lo que me propongo, en verdad, volvería a intentarlo una y otra vez hasta lograrlo" [E-ADPPV-MET/OB21]. Los Casos 2, 3, 4 y 6 comentan que no tienen claro ni han pensado sus objetivos, confesando que planificar su proyecto vital es una tarea ardua. El Caso 5 tiene claras cuáles son sus metas de futuro, pero no sabe cómo lograrlas. Las complejas trayectorias de vida de los casos estudiados muestran lo injusto que es estar condicionado desde la infancia por un contexto que no ayuda al desarrollo social y al crecimiento personal.

\section{Discusión y conclusiones}

El análisis de los casos permite comprender los múltiples impedimentos a los que se enfrentan los menores de colectivos desfavorecidos al construir sus proyectos vitales. La complejidad y multidimensionalidad del concepto de exclusión social (Parrilla et al., 2010) nos ha llevado a seleccionar casos que reflejan trayectorias de jóvenes con precarias economías familiares y/o influenciados negativamente por las características contextuales. Los seis menores tienen: 1) expectativas académicas y profesionales limitadas y difusas, 2) escasas metas u objetivos autoimpuestos, 3) vago razonamiento en la toma de decisiones, 4) conducta social conflictiva, 5) desempeño de un rol social equivocado y 6) impulsividad a la hora de actuar.

Los casos analizados en este estudio son un ejemplo de las dificultades de los adolescentes en riesgo de exclusión para proyectar su futuro, sobre todo cuando las condiciones de vida han sido y son inadecuadas para su desarrollo intelectual, emocional y social. Los elementos intra e interpersonales determinan el proceso de elaboración del proyecto personal de vida e influyen en el procesamiento racional de 
la toma de decisiones (D'Angelo, 2000). El déficit en inteligencia emocional y habilidades sociales complica la tarea de construir un itinerario profesional y personal ajustado a las necesidades e intereses propios.

Los proyectos personales de vida de los adolescentes en riesgo son construidos desde la inadaptación al sistema y sobre una base socioemocional deficiente. Los resultados de nuestro estudio evidencian que el proceso de toma de decisiones y de construcción del proyecto personal de vida depende de la forma de procesar cognitivamente la información; dicho proceso está influenciado por el contexto y las capacidades socioemocionales de los adolescentes. Las familias apuestan por la continuidad de los estudios como vía de salida de los procesos de exclusión y marginalidad y espera que la institución escolar ofrezca otros modelos socioeducativos para la configuración del proyecto vital de los adolescentes. Sin embargo, es complicado que estos se mantengan al margen de los modelos sociofamiliares de referencia (Santana et al., 2018).

En la construcción de los proyectos vitales de los menores inciden factores como la acción de agentes externos (sociales, familiares y educativos) y la autoexclusión, generada bien por decisión propia o por su inadaptación. Para atender las necesidades de los menores en riesgo de exclusión social habría que analizar su contexto vital e identificar los factores de riesgo y de protección presentes en sus realidades. El análisis y la comprensión del contexto socioeconómico de los adolescentes en conflicto es relevante para entender su comportamiento (Ducca-Cisneros, 2018; García et al., 2016; González-Cubillán, 2009; Melendro, 2011; Melendro et al., 2014; Pérez et al., 2016; Pérez \& Melendro, 2016; Rodríguez et al., 2016).

Del estudio realizado se derivan algunas implicaciones para la práctica educativa y orientadora. Las escasas estrategias y destrezas socioemocionales de los adolescentes en riesgo de exclusión social plantean la urgente necesidad de trabajar y cambiar esa realidad. Los servicios de orientación, de manera coordinada con los agentes educativos de los centros de día, deben diseñar programas de atención/apoyo para: 1) prevenir conductas intra e interpersonales conflictivas; 2) dotar a estos adolescentes de estrategias para afrontar las múltiples situaciones de riesgo en las que se ven inmersos; 3) ofrecer a las familiar pautas educativas adecuadas a las casuística de estos adolescentes (Caravantes et al., 2017; Castel, 2014; Lomelí-Parga et al., 2016; Melendro et al., 2017).

La investigación abre nuevas líneas de trabajo sobre la influencia del contexto sociofamiliar en la construcción del proyecto vital de adolescentes en riego para comprender sus comportamientos y actitudes (Ballester et al., 2016; Melendro et al., 2014). Nos planteamos las siguientes cuestiones: ¿de qué forma se puede ayudar a los jóvenes en riesgo de exclusión a superar las adversidades sociales y personales que les impiden desarrollar un proyecto vital consistente?, ¿cómo potenciar la capacidad para tomar decisiones teniendo en cuenta las experiencias de vida de los menores en riesgo de exclusión?, ¿de qué forma los programas para la mejora de las capacidades inter e intrapersonales contribuyen a la configuración de proyectos vitales sólidos y a la madurez de los jóvenes en riesgo?, ¿qué proyectos de vida se plantean los jóvenes en riesgo de exclusión que no estén acogidos a centros de protección?

Las limitaciones principales de este estudio están relacionadas con el número de casos analizados, sin embargo, la descripción de los mismos permite comprender y 
visibilizar la realidad socioeducativa de adolescentes en riesgo. Para poder confirmar los hallazgos de la investigación, sería conveniente llevar a cabo un estudio de carácter longitudinal con un mayor número de participantes, y hacer un seguimiento de las trayectorias de vida de dichos adolescentes.

\section{Referencias}

Álvarez, M., Bisquerra, R., Espín, L. y Rodríguez, S. (2007). La madurez para la Carrera en la Educación Secundaria. Sevilla, España: EOS.

Anguera, M. T., Blanco-Villaseñor, A., Losada, J. L., Sánchez-Algarra, P. y Onwuegbuzie, A. J. (2018). Revisiting the difference between mixed methods and multimethods: Is it all in the name? Quality \& Quantity, 52(6) 2757-2770. doi: https://doi.org/10.1007/ s11135-018-0700-2

Ballester, L., Caride, J. L., Melendro, M. y Montserrat, C. (2016). Jóvenes que construyen futuros: de la exclusión a la inclusión social. Universidad de Santiago de Compostela: Servicio de Publicaciones e Intercambio Científico

Bautista-Cerro, M. J. y Melendro, M. (2011). Competencias para la intervención socioeducativa con jóvenes en dificultad social. Educación XXI, 14(1), 179-200. Recuperado de http://revistas.uned.es/index.php/educacionXX1/article/view/268/224

Bendit, R. y Hahn-Bleibtreu, M. (2008). Youth transitions: processes of social inclusion and patterns of vulnerability in a globalised world. Budrich: Opladen [u.a].

Bendit, R. y Stokes, D. (2004). Jóvenes en situación de desventaja social: políticas de transición entre la construcción social y las necesidades de una juventud vulnerable. Revista de Estudios de Juventud, 65, 11-29.

Boutinet, A. (2002). Antropologia do projeto. Porto Alegre, Brasil: Artmed.

Caravantes, G. M., Climent López, M. y Masiá R. (2017). Los efectos de barrio en la adolescencia: una comparativa entre el barrio del Xenillet y el barrio de La Coma desde la perspectiva comunitaria. Revista Internacional de Trabajo Social y Bienestar, 6, 5-15.

Carbonero, M. y Merino, E. (2003). La escala de autoeficacia vocacional: desarrollo, análisis y aplicaciones del instrumento. Revista de Psicodidáctica, 14, 99-114.

Castel, R. (2014). Los riesgos de exclusión social en un contexto de incertidumbre. Revista Internacional de Sociología, 72(extra1), 15-24. doi:10.3989/ris.2013.03.18

D'Angelo, O. (2000). Proyecto de vida como categoría básica de interpretación de la identidad individual y social. Revista Cubana de Psicología, 17(3), 270-275.

Maree, J.G. (2018). Promoting Career Development and Life Design in the Early Years of a Person's Life. Early Child Development and Care, 188(4), 425-436. http://dx.doi. org/10.1080/03004430.2017.1345892

Du Bois-Reymond, M. y López, A. (2004). Transiciones tipo yo-yo y trayectorias fallidas: hacia las políticas integradas de transición para jóvenes europeos. Revista de Estudios de juventud, (65), 11-29.

Ducca-Cisneros, L. V. (2018). Elegir en tiempos revueltos: orientación vocacional y adolescentes "en riesgo" de exclusión social. Trabajo Social Global, 8(14), 125-146. doi: 10.30827/tsg-gsw.v8i14.7241 
Figuera, P., Dorio, I. y Forner, À. (2003). Las competencias académicas previas y el apoyo familiar en la transición a la universidad. Revista de Investigación Educativa, 21 (2), 349-369.

Freire, P. (1998). La educación como práctica de la libertad. Madrid, España: Siglo XXI.

García, J. L., Quintanal, J. y Cuenca, M. E. (2016). Análisis de la percepción que tienen los profesores y las familias de los valores en los jóvenes en vulnerabilidad social. Revista Española de Pedagogía, 263, 91-108.

González-Cubillán, L. (2009). El proyecto de vida, estrategia para enfrentar la adversidad: estudio de casos. Encuentros multidisciplinares, 11(31), 21-26.

Lomelí-Parga, A., López-Padilla, M. y Valenzuela-González, J. (2016). Autoestima, motivación e inteligencia emocional: Tres factores influyentes en el diseño exitoso de un proyecto de vida de jóvenes estudiantes de educación media. Revista Electrónica Educare, 20(2), 1-22. doi: http://dx.doi.org/10.15359/ree.20-2.4

Melendro, M. (2011). El tránsito a la vida adulta de los jóvenes en dificultad social. Zerbitzuan, 49, 93-106. doi:10.5569/1134-7147.49.08

Melendro, M. (2014). Transitar a la vida adulta cuando se es joven y vulnerable: estrategias de actuación en una sociedad en crisis. Metamorfosis. Revista del Centro Reina Sofía sobre Adolescencia y Juventud, 1, 37-54.

Melendro, M., De-Juanas, A. Rodríguez, A. E. (2017). Déficits en la intervención socioeducativa con familias de adolescentes en riesgo de exclusión. Bordón, 69(1), 123-138. doi: 10.13042/Bordon.2016.48596

Melendro, M., Cruz, L., Iglesias, A. y Montserrat, C. (2014). Estrategias eficaces de intervención socioeducativa con adolescentes en riesgo de exclusión. Madrid: UNED

Parrilla, Á., Gallego, C. y Moriña, A. (2010). El complicado tránsito a la vida activa de jóvenes en riesgo de exclusión: una perspectiva biográfica. Revista de Educación, (351), 211-233.

Pérez, G. y Melendro, M. (2016). Ocio, formación y empleo de los jóvenes en dificultad social. Revista Española de Pedagogía, 263, 5-11.

Pérez, G., Poza, F. y Fernández, A. (2016). Criterios para una intervención de calidad con jóvenes en dificultad social. Revista Española de Pedagogía, 263, 51-69.

Rodríguez, A.E., de Juanas, A. y González A.L. (2016) Atribuciones de los jóvenes en situación de vulnerabilidad social sobre los beneficios del estudio y la inserción laboral. Revista Española de Pedagogía, 263, 109-126.

Saldaña, J. (2009). El manual de codificación para investigadores cualitativos. Londres: Sage.

Santana-Vega, L. E. (2015). Orientación educativa e intervención psicopedagógica. Madrid: Pirámide.

Santana Vega, L.E.; Alonso Bello, E.; Feliciano García, L. (2018). Trayectorias laborales y competencias de empleabilidad de jóvenes nacionales e inmigrantes en riesgo de exclusión social. Revista Complutense de Educación, 29(2), 355-369. doi: http://dx.doi. org/10.5209/RCED.52444

Santana-Vega, L. E., Feliciano-García, L. y Santana-Lorenzo, A. (2013). Madurez y autoeficacia vocacional en $3^{\circ}$ y $4^{\circ}$ de la ESO, Bachillerato y Ciclos Formativos. Una perspectiva de género. Revista Española de Orientación y Psicopedagogía, 24(3), 8-26. 
Santana-Vega, L., Feliciano-García, L. y Jiménez, A. (2016). Apoyo familiar percibido y proyecto de vida del alumnado inmigrante de educación secundaria. Revista Educación, 372, 35-58. doi: http://dx.doi.org/10.4438/1988-592X-RE-2015-372-314

Velaz de Medrano, C. (2005). Medidas para prevenir el rechazo escolar y evitar la exclusión social desde un enfoque democrático y comunitario de la atención a la diversidad. En VV.AA. (Eds.), Educación para la ciudadanía (pp. 90-108). Madrid, España: Atlántida.

Fecha de recepción: 24 de mayo de 2018.

Fecha de revisión: 20 de junio de 2018.

Fecha de aceptación: 4 de julio de 2019 
